\title{
A Partially Penalised Immersed Finite Element Method for Elliptic Interface Problems with Non-Homogeneous Jump Conditions
}

\author{
Haifeng $\mathrm{Ji}^{1}$, Qian Zhang ${ }^{2, *}$, Qiuliang Wang ${ }^{3}$ and Yifan Xie ${ }^{4}$ \\ ${ }^{1}$ School of Science, Nanjing University of Posts and Telecommunications, Nanjing \\ 210023, Jiangsu, China. \\ ${ }^{2}$ Institute of Information Technology, Nanjing University of Chinese Medicine, \\ Nanjing 210023, Jiangsu, China. \\ ${ }^{3}$ School of Mathematics and Statistics, Shangqiu Normal University, Shangqiu \\ 476000, Henan, China. \\ ${ }^{4}$ School of Earth Sciences and Engineering, Nanjing University, Nanjing 210023, \\ China.
}

Received 16 February 2017; Accepted (in revised version) 7 July 2017.

\begin{abstract}
A partially penalised immersed finite element method for interface problems with discontinuous coefficients and non-homogeneous jump conditions based on unfitted meshes independent of the interface is proposed. The arising systems of linear equations have symmetric positive definite matrices which allows the use of fast solvers and existing codes. Optimal error estimates in an energy norm are derived. Numerical examples demonstrate the efficiency of the method.
\end{abstract}

AMS subject classifications: 65N15, 65N30, 35J60

Key words: Immersed finite element method, interface problem, Cartesian mesh, non-homogeneous jump condition, closest-point projection.

\section{Introduction}

We consider an immersed interface finite element method to solve the elliptic interface problem

$$
\begin{array}{ll}
-\nabla \cdot \beta(x, y) \nabla u(x, y)=f(x, y), & x \in \Omega \backslash \Gamma, \\
u(x, y)=0, & x \in \partial \Omega,
\end{array}
$$

together with the following non-homogeneous jump conditions across the interface $\Gamma$ :

$$
\begin{aligned}
& {[u]_{\Gamma}=u^{+}-u^{-}=w,} \\
& {\left[\beta \frac{\partial u}{\partial \mathbf{n}}\right]_{\Gamma}=\beta^{+} \nabla u^{+} \cdot \mathbf{n}-\beta^{-} \nabla u^{-} \cdot \mathbf{n}=Q,}
\end{aligned}
$$

*Corresponding author. Email address: zq19880125@163.com (Q. Zhang) 


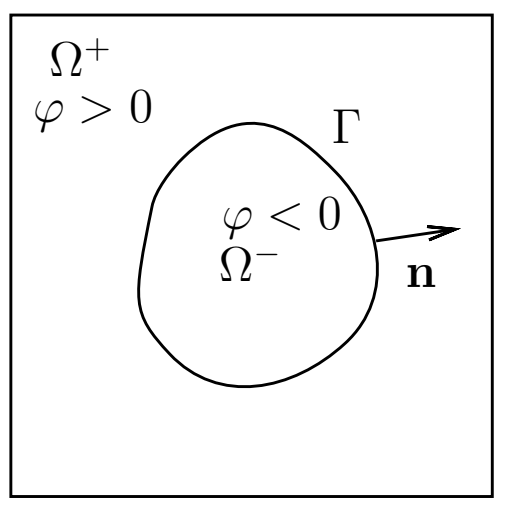

Figure 1: The geometry of an interface problem.

where $u^{ \pm}(x, y)=\left.u(x, y)\right|_{\Omega^{ \pm}}$and $\mathbf{n}$ is a unit normal to the interface pointing from $\Omega^{-}$to $\Omega^{+}$. In Fig. 1 for an illustration, without loss of generality, we assume that $\Omega \subset \mathbb{R}^{2}$ is a rectangular domain, the interface is a closed and smooth curve separating $\Omega$ into two subdomains $\Omega^{-}, \Omega^{+}$such that $\Omega^{-}$lies strictly inside $\Omega$, and the coefficient $\beta(x, y)$ is a positive and piecewise constant function - i.e.

$$
\beta(x, y)=\left\{\begin{array}{ll}
\beta^{-}, & (x, y) \in \Omega^{-}, \\
\beta^{+}, & (x, y) \in \Omega^{+},
\end{array} \text {and } \quad \beta^{ \pm}>0 .\right.
$$

The homogeneous Dirichlet boundary condition $u=0$ is used just for convenience in the theoretical analysis, since we focus on the interface. Indeed, other boundary conditions (e.g. non-homogeneous boundary conditions or Neumann boundary conditions) can be treated using standard finite element techniques. As in the classical level set method, the interface $\Gamma$ is implicitly defined as the zero level set of a smooth function $\varphi(x, y)$ satisfying

$$
\varphi(x, y) \begin{cases}<0, & (x, y) \in \Omega^{-} \\ =0, & (x, y) \in \Gamma, \\ >0, & (x, y) \in \Omega^{+}\end{cases}
$$

The unit normal $\mathbf{n}$ to the interface $\Gamma$ pointing from $\Omega^{-}$to $\Omega^{+}$is then $\mathbf{n}=\nabla \varphi /\|\nabla \varphi\|_{2}$. It is notable that the method we propose is not restricted to the level set representation of the interface, for it also works for a parametric representation of the interface - cf. Section 2.4.

The interface problem (1.1)-(1.4) arises in many important scientific and engineering applications. For example, in electrostatic field computations this interface problem involves interface data $Q$ referring to surface charge density [14]. For the computation of the temperature in composite media with thermal contact resistance effect, the solution may have jumps across the interface - i.e., $w \neq 0$. We are interested in solving such an interface problem by a finite element (FE) method based on unfitted meshes independent of the interface. Numerical methods using such meshes have some advantages compared with body fitted meshes, including the attractive ease in handling problems with moving interfaces. An early numerical method that used an unfitted mesh is 
Peskin's immersed boundary (IB) method [32], where the interface jump conditions are treated as singular sources, and since then various methods using unfitted meshes have been developed - e.g. the immersed interface method $[18,20]$, the immersed finite element/volume method $[1,10,13,15,22,25,26,30,31]$, the augmented finite difference/element method $[16,17,19,23]$, the extended finite element method [6], the unfitted Nitsche's finite element method [8,29,35,36], the kernel-free boundary integral method [37-39], the virtual node method [2,11], the ghost fluid method [27,28], the coupling interface method [4,34], and the matched interface and boundary method [40,41]. Of these methods, the immersed finite element (IFE) methods have attracted considerable attention since they are relatively simple and convenient in several aspects. First of all, the degrees of freedom remain the same as that of the traditional FE method for problems with no interface, whereas the extended or unfitted Nitsche's FE methods require extra degrees of freedom on interface elements to capture jumps across the interface. Secondly, the stiffness matrix of an IFE method is computed element-by-element just like in the traditional FE method; and one only needs to modify the shape function on interface elements, so the method can be incorporated readily into existing FE software packages. Thirdly, the IFE method reduces to the traditional FE method when discontinuities and interfaces disappear. Finally, the solution obtained by the IFE method has optimal convergence, as if there is no interface.

However, most IFE methods are designed for homogeneous jump conditions. For interface problems with both discontinuous coefficients and non-homogeneous jump conditions, Gong et al. [7] developed a source removal technique that essentially moved the non-homogeneous terms to the right-hand side using the level set function. Subsequently, a similar technique was proposed in Ref. [5] to solve the interface problem in which the coefficient was a constant without a jump. He et al. [9] developed an IFE method by enriching the classical IFE spaces locally on interface elements to capture the non-homogeneous flux jump condition with $w=0$. Using similar ideas, Chang \& Kwak [3] proposed an approach by constructing a bubble function that satisfied the same non-homogeneous jump conditions as the solution in a certain sense. Hou \& Liu [12] proposed a Petrov-Galerkin finite element method that can also deal with non-homogeneous jump conditions, where the stiffness matrix is non-symmetric since the solution function is taken from the classical IFE space while the testing function is from the traditional linear conforming finite element space. These IFE methods for interface problems with both discontinuous coefficients and non-homogeneous jump conditions are shown to be effective, but the convergence analysis is extremely difficult and is still an open question.

Recently, Lin et al. [24] proposed a partially penalised IFE method for elliptic interface problems with discontinuous coefficients but homogeneous jump conditions. This method introduces extra terms only at interface edges, to handle discontinuities of functions in classical IFE spaces. With these modifications, the optimal error estimates in an energy norm were obtained and the convergence orders in both the $H^{1}$ and $L^{2}$ norms do not deteriorate when the mesh becomes finer, which is sometimes a shortcoming of the classical IFE methods. Here we develop a partially penalised IFE method for solving the interface problem (1.1)-(1.4) with both discontinuous coefficients and non-homogeneous jump con- 


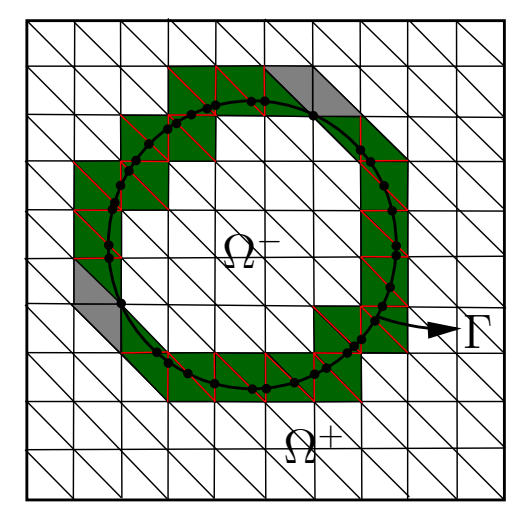

Figure 2: An unfitted mesh. Gray: $\mathscr{T}_{h}^{d} ;$ Dark green: $\mathscr{T}_{h}^{\text {int }} ;$ Red: $\mathscr{E}_{h}^{\text {int }}$.

ditions. The idea to deal with the non-homogeneous jumps across the interface originally comes from Ref. [7,23], where a function satisfying the same non-homogeneous jumps is constructed by using the level set representation of the interface and extensions of the interface data. The correction function is non-polynomial and defined on the supports of the basis functions the interface cuts through, so the interface data need to be extended to the tube of $2 h$ distance of the interface [7]. Moreover, the gradient or Laplacian of the non-polynomial correction function needs to be computed numerically, which also makes the method harder to implement. In contrast, the correction function in our method is piecewise linear and constructed locally on interface elements, and the interface data only need to be extended to the vertices of the interface elements in the tube of $h$ distance of the interface. The extension is obtained by using a closest-point projection. We prove the optimal error estimate in an energy norm, and present numerical examples to show the performance of the method. To the best of our knowledge, this is the first IFE method that proves to be convergent optimally in an energy norm for an elliptic interface problem with both discontinuous coefficients and non-homogeneous jump conditions.

This paper is organized as follows. In Section 2, we review the classical IFE space, construct a correction function $u_{h}^{J}$, and introduce a partially penalised IFE method. Section 3 deals with optimal error estimates in an energy norm. Numerical examples, illustrating theoretical results, are discussed in Section 4. Our concluding remarks are in Section 5.

\section{Finite Element Method}

In order to introduce the partially penalised IFE method for the interface problem (1.1)(1.4), let us assume that $\Omega$ is a rectangular domain with a Cartesian mesh - cf. Fig. 2. Splitting $\Omega$ into uniform rectangles with the side lengths of $\Delta x$ and $\Delta y$ and dividing the rectangles by parallel diagonals, one obtains a triangulation $\mathscr{T}_{h}$ with the mesh size $h=$ $\sqrt{(\Delta x)^{2}+(\Delta y)^{2}}$. We also assume that $h$ can be chosen such that each triangle has either at most two common points with $\Gamma$ or one of its edges belongs to $\Gamma$. Moreover, if there are exactly two common points, they are located on different closed edges of the triangle. 
Let $\mathscr{N}_{h}$ refer to the set of all vertices $\left(x_{i}, y_{i}\right) \in \bar{\Omega}$ of the triangular elements and let

$$
\mathscr{N}_{h}^{i}:=\mathscr{N}_{h} \cap \Omega, \quad \mathscr{N}_{h}^{b}:=\mathscr{N}_{h} \cap \partial \Omega, \quad \mathscr{N}_{h}^{s}:=\mathscr{N}_{h} \cap \Gamma .
$$

An element $T$ is called the interface element if $\Gamma$ crosses the interior of $T$, otherwise it is refereed to as a non-interface element. The sets of all interface and non-interface elements are denoted by $\mathscr{T}_{h}^{\text {int }}$ and $\mathscr{T}_{h}^{\text {non }}$, respectively. We also consider the set

$$
\mathscr{T}_{h}^{d}=\left\{T \in \mathscr{T}_{h}^{\text {non }}: T \subset \Omega^{+}, \Gamma \cap \partial T \in \mathscr{N}_{h}^{s} \text { or an edge of } T \text { is a subset of } \Gamma\right\},
$$

and for $t \in \Gamma$ we define $u(t):=\lim _{\substack{\tau \rightarrow t \\ \tau \in \Omega^{-}}} u(\tau)$.

Similar to the set of interior open edges $\mathscr{E}_{h}$ in $\mathscr{T}_{h}$, let us introduce the set of interface edges

$$
\mathscr{E}_{h}^{i n t}:=\left\{e \in \mathscr{E}_{h}: e \cap \Gamma \neq \emptyset\right\},
$$

so that $\mathscr{E}_{h}^{\text {non }}=\mathscr{E}_{h} \backslash \mathscr{E}_{h}^{\text {int }}$, where $\mathscr{E}_{h}^{\text {non }}$ denotes the set of non-interface edges - cf. Fig. 2 showing the sets $\mathscr{T}_{h}^{\text {int }}, \mathscr{E}_{h}^{\text {int }}$ and $\mathscr{T}_{h}^{d}$.

\subsection{Classical IFE space for homogeneous jump conditions}

Let us briefly describe the classical IFE space - cf. [22]. For each non-interface element the functions of IFE are linear, otherwise they are piecewise linear. We consider a typical interface element $\triangle A B C$ of the IFE space shown in Fig. 3. The line segment $\overline{D E}$ splits $T$ into two parts $T_{h}^{+}$and $T_{h}^{-}$. Let $\mathbf{n}_{h}$ and $\mathbf{t}_{h}$ be, respectively, the unit normal and the unit tangential vectors of $\overline{D E}$. For this element $T$, an IFE function has the form

$$
v_{h}(x, y)= \begin{cases}v_{h}^{+}(x, y)=a^{+}+b^{+} x+c^{+} y, & (x, y) \in T_{h}^{+}, \\ v_{h}^{-}(x, y)=a^{-}+b^{-} x+c^{-} y, & (x, y) \in T_{h}^{-},\end{cases}
$$

where coefficients $a^{ \pm}, b^{ \pm}, c^{ \pm}$are specified in order to satisfy the relations

$$
\begin{aligned}
& v_{h}\left(x_{A}, y_{A}\right)=V_{1}, \quad v_{h}\left(x_{B}, y_{B}\right)=V_{2}, \quad v_{h}\left(x_{C}, y_{C}\right)=V_{3}, \\
& v_{h}^{+}\left(x_{D}, y_{D}\right)=v_{h}^{-}\left(x_{D}, y_{D}\right), \quad v_{h}^{+}\left(x_{E}, y_{E}\right)=v_{h}^{-}\left(x_{E}, y_{E}\right), \quad \beta^{+} \nabla v_{h}^{+} \cdot \mathbf{n}_{h}=\beta^{-} \nabla v_{h}^{-} \cdot \mathbf{n}_{h},
\end{aligned}
$$

and $V_{i}, i=1,2,3$ are the nodal variables. Let us emphasise that functions (2.1), containing six unknowns, shall satisfy six conditions (2.2)-(2.3). It was shown in Ref. [22] that $V_{i}$, $i=1,2,3$ uniquely determine a piecewise linear function.

Let $V_{h}^{I F E}$ stand for an IFE space consisting of all functions $v_{h}$ such that

1. For any $T \in \mathscr{T}_{h}^{\text {non }}$, the restriction $v_{h}$ on $T$ is a linear function;

2. For any $T \in \mathscr{T}_{h}^{\text {int }}$, the restriction $v_{h}$ on $T$ is a piecewise linear function defined by Eqs. (2.1)-(2.3);

3. For any nodal point $\left(x_{i}, y_{i}\right) \in \mathscr{N}_{h}$, the function $v_{h}$ is continuous;

4. For any nodal point $\left(x_{i}, y_{i}\right) \in \mathscr{N}_{h}^{b}$, the condition $v_{h}\left(x_{i}, y_{i}\right)=0$ holds. 


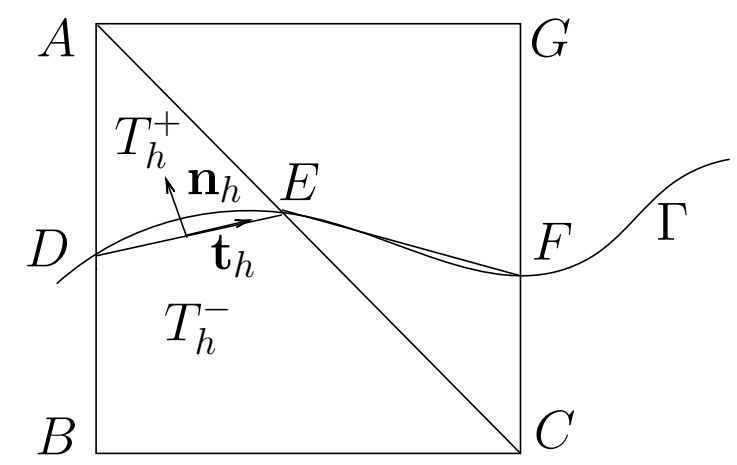

Figure 3: A typical interface element and a neighboring element.

Let $V_{h}^{L}$ be the standard linear conforming finite element space. It is clear that the IFE space $V_{h}^{I F E}$ is a modification of the space $V_{h}^{L}$ for discontinuous coefficients $\beta(x, y)$. For the same mesh, these two spaces coincide if $\beta^{+}=\beta^{-}$. It is well known that on the edges $\mathscr{E}_{h}^{\text {int }}$, IFE functions can be discontinuous. For example, for given $v_{h}\left(x_{A}, y_{A}\right), v_{h}\left(x_{B}, y_{B}\right), v_{h}\left(x_{C}, y_{C}\right)$ and $v_{h}\left(x_{G}, y_{G}\right)$, the restrictions $\left.v_{h}\right|_{\triangle A B C}$ and $\left.v_{h}\right|_{\triangle A C G}$ of $v_{h}$ on the triangles $\triangle A B C$ and $\triangle A C G$ are independently determined and the values of $v_{h}$ at the point $E$ may not coincide cf. Fig. 3, so that $v_{h}(x, y)$ can have a discontinuity on $A C$.

For a function $v(x, y)$, let $I_{h}^{I F E} v \in V_{h}^{I F E}$ denote the IFE interpolation of $v$ such that $I_{h}^{I F E} v\left(x_{i}, y_{i}\right)=v\left(x_{i}, y_{i}\right)$ for all nodal points $\left(x_{i}, y_{i}\right) \in \mathscr{N}_{h}$. The IFE space $V_{h}^{I F E}$ has an optimal approximation property for solutions with homogeneous jump conditions. More exactly, the following result holds.

Lemma 2.1 (cf. Li et al. [21]). If $v \in H^{2}\left(\Omega^{+} \cup \Omega^{-}\right)$satisfies the homogeneous jump conditions $[v]_{\Gamma}=0$ and $[\beta(\partial v / \partial \mathbf{n})]_{\Gamma}=0$, then there is a constant $C>0$, independent of $h$, such that for all $T \in \mathscr{T}_{h}^{\text {int }}$ one has

$$
\begin{aligned}
& \left\|v-I_{h}^{I F E} v\right\|_{L^{2}(T)} \leq C h^{2}\|v\|_{H^{2}\left(T^{+} \cup T^{-}\right)}, \\
& \left\|v-I_{h}^{I F E} v\right\|_{H^{1}(T)} \leq C h\|v\|_{H^{2}\left(T^{+} \cup T^{-}\right)},
\end{aligned}
$$

where $T^{+}=T \cap \Omega^{+}$and $T^{-}=T \cap \Omega^{-}$.

\subsection{A correction function for non-homogeneous jumps}

Fix a $\rho>0$, and let $N(\Gamma, \rho)$ be the neighbourhood of $\Gamma$,

$$
N(\Gamma, \rho):=\{(x, y) \in \Omega: \operatorname{dist}((x, y), \Gamma) \leq \rho\},
$$

where $\operatorname{dist}((x, y), \Gamma)$ is the distance from $(x, y)$ to $\Gamma$. If $\rho$ is sufficiently smaller than the curvature of the interface, then for any $(x, y) \in N(\Gamma, \rho)$ there is a unique closest-point projection $(X, Y)$ of $(x, y)$ on $\Gamma$ such that

$$
\|(x, y)-(X, Y)\|_{2}=\operatorname{dist}((x, y), \Gamma) .
$$


It is clear that the information related to the interface $\Gamma$ is governed by the level set function $\varphi(x)$ considered in a neighborhood of the interface. Let us now consider the signed distance function

$$
d(x, y):=\left\{\begin{array}{cl}
-\operatorname{dist}((x, y), \Gamma) & \text { if }(x, y) \in \Omega^{-} \cap N(\Gamma, \rho), \\
0 & \text { if }(x, y) \in \Gamma, \\
\operatorname{dist}((x, y), \Gamma) & \text { if }(x, y) \in \Omega^{+} \cap N(\Gamma, \rho) .
\end{array}\right.
$$

If a function $u$ is evaluated on the interface $\Gamma$, its extension $u^{e}$ to a neighborhood $N(\Gamma, \rho)$ of $\Gamma$ is defined by

$$
u^{e}(x, y):=u(X, Y), \quad(x, y) \in N(\Gamma, \rho),
$$

where $(X, Y)$ is the closest-point projection of $(x, y)$ on the interface $\Gamma$.

Given interface functions $w$ and $Q$, let us define the functions $\widetilde{u}(x, y)$ and $\widehat{u}(x, y)$ by

$$
\begin{aligned}
& \widetilde{u}(x, y):=w^{e}(x, y)+\frac{Q^{e}(x, y)}{\beta^{+}} d(x, y), \quad(x, y) \in N(\Gamma, \rho), \\
& \widehat{u}(x, y):=H(\varphi(x, y)) \widetilde{u}(x, y), \quad(x, y) \in N(\Gamma, \rho),
\end{aligned}
$$

with the Heaviside function

$$
H(s)= \begin{cases}0 & \text { if } s \leq 0 \\ 1 & \text { if } s>0\end{cases}
$$

If $\Gamma$ and the interface functions $w$ and $Q$ are sufficiently smooth, then the function $\widetilde{u}$ is also sufficiently smooth and (2.4), (2.5) yield the relations

$$
\begin{aligned}
& \left.\widetilde{u}\right|_{\Gamma}=w \\
& \left.\frac{\partial \widetilde{u}}{\partial \mathbf{n}}\right|_{\Gamma}=\left.\frac{\partial w^{e}}{\partial \mathbf{n}}\right|_{\Gamma}+\left.\frac{d(x, y)}{\beta^{+}} \frac{\partial Q^{e}}{\partial \mathbf{n}}\right|_{\Gamma}+\left.\frac{Q^{e}}{\beta^{+}} \frac{\partial d(x, y)}{\partial \mathbf{n}}\right|_{\Gamma}=\frac{Q}{\beta^{+}} .
\end{aligned}
$$

Moreover, it is easily seen that the function

$$
u^{h o m}(x, y):=u(x, y)-\widehat{u}(x, y), \quad(x, y) \in N(\Gamma, \rho),
$$

satisfies the homogeneous interface jump conditions - i.e.

$$
\left[u^{\text {hom }}\right]_{\Gamma}=0, \quad\left[\beta \frac{\partial u^{\text {hom }}}{\partial \mathbf{n}}\right]_{\Gamma}=0 .
$$

Remark 2.1. The previous considerations deal with the construction of a function $\widetilde{u}$ satisfying the conditions

$$
\widetilde{u}(x, y)=w(x, y), \quad \beta^{+} \frac{\partial \widetilde{u}}{\partial \mathbf{n}}(x, y)=Q(x, y), \quad(x, y) \in \Gamma
$$


This can also be done by setting $\Omega_{\rho}^{ \pm}:=\Omega^{ \pm} \cap N(\Gamma, \rho)$ and determining the required function $\tilde{u} \in H^{2}\left(\Omega_{\rho}\right)$ from the biharmonic problem

$$
\begin{aligned}
& \Delta^{2} \widetilde{u}^{ \pm}(x, y)=0 \quad(x, y) \in \Omega_{\rho}^{ \pm}, \\
& \widetilde{u}^{ \pm}(x, y)=w(x, y), \quad \beta^{+} \frac{\partial \widetilde{u}^{ \pm}}{\partial \mathbf{n}}(x, y)=Q \quad(x, y) \in \Gamma, \\
& \widetilde{u}^{ \pm}(x, y)=0, \quad \frac{\partial \widetilde{u}^{ \pm}}{\partial \mathbf{n}}=0 \quad(x, y) \in \partial \Omega_{\rho}^{ \pm} \backslash \Gamma .
\end{aligned}
$$

Although for $\Gamma \in C^{2}$ the problem (2.10) has a unique solution $\widetilde{u} \in H^{2}\left(\Omega_{\rho}\right)$, its derivation meets considerable difficulties and we do not pursue this idea here.

In order to find a correction function which would handle the jumps in non-homogeneous interface conditions and be suitable for our numerical method, we use the interpolation operator $I_{h}^{L}$ - viz. if $v$ is a piecewise continuous function such that $\left.v\right|_{\Omega^{+}}=v^{+} \in C\left(\Omega^{+}\right)$and $\left.v\right|_{\overline{\Omega^{-}}}=v^{-} \in C\left(\overline{\Omega^{-}}\right)$, then $I_{h}^{L} v \in V_{h}^{L}$ is defined by

$$
I_{h}^{L} v\left(x_{i}, y_{i}\right):=v\left(x_{i}, y_{i}\right), \quad\left(x_{i}, y_{i}\right) \in \mathscr{N}_{h} .
$$

Let

$$
\Omega_{\Gamma}:=\bigcup_{T \in \mathscr{T}_{h}^{\text {int }} \cup \mathscr{T}_{h}^{d}} T
$$

Then

$$
u_{h}^{J}(x, y):= \begin{cases}H(\varphi) I_{h}^{L} \widetilde{u}-I_{h}^{I F E}(H(\varphi) \widetilde{u}) & \text { if }(x, y) \in \Omega_{\Gamma}, \\ 0 & \text { otherwise },\end{cases}
$$

is the correction function required.

We note that for $h<\rho$ one has $\Omega_{\Gamma} \subset N(\Gamma, \rho)$. Moreover, the function $u_{h}^{J}$ is piecewise polynomial with support $\Omega_{\Gamma}$ and is completely determined by the values of $\widetilde{u}$ at the vertices of the triangles in $\mathscr{T}_{h}^{\text {int }}$ and $\mathscr{T}_{h}^{d}$. It is easily seen that all discontinuities of $u_{h}^{J}$ are located along the interface $\Gamma$.

Considering another function

$$
u^{J}(x, y)= \begin{cases}\widehat{u}-I_{h}^{I F E} \widehat{u}=H(\varphi) \widetilde{u}-I_{h}^{I F E}(H(\varphi) \widetilde{u}) & \text { if }(x, y) \in \Omega_{\Gamma}, \\ 0 & \text { otherwise },\end{cases}
$$

we note that it is non-polynomial and satisfies the interface jump conditions exactly but it may have a discontinuity on the boundary of $\Omega_{\Gamma}$.

The following lemma describes approximation properties of the correction function $u_{h}^{J}$.

Lemma 2.2. Assume that the interface $\Gamma$ and the interface functions $w$ and $Q$ are such that the function $\tilde{u}$ satisfying the conditions (2.9), belongs to the space $H^{2}(N(\Gamma, \rho))$. Then for any 
$u \in H^{2}\left(\Omega^{+} \cup \Omega^{-}\right)$satisfying (1.3)-(1.4), there is a constant $C>0$, independent of $h$, such that for any $h<\rho$ the inequalities

$$
\begin{aligned}
& \left\|u-I_{h}^{I F E} u-u_{h}^{J}\right\|_{L^{2}(\Omega)} \leq C h^{2}\left(\|u\|_{H^{2}\left(\Omega^{+} \cup \Omega^{-}\right)}+\|\widetilde{u}\|_{H^{2}\left(\Omega_{\Gamma}\right)}\right) \\
& \left(\sum_{T \in \mathscr{T}_{h}}\left\|u-I_{h}^{I F E} u-u_{h}^{J}\right\|_{H^{1}(T)}^{2}\right)^{1 / 2} \leq C h\left(\|u\|_{H^{2}\left(\Omega^{+} \cup \Omega^{-}\right)}+\|\widetilde{u}\|_{H^{2}\left(\Omega_{\Gamma}\right)}\right)
\end{aligned}
$$

hold.

Proof. If $T \in \mathscr{T}_{h}^{\text {int }} \cup \mathscr{T}_{h}^{d}$, then (2.11)-(2.12) yield

$$
\begin{aligned}
u-I_{h}^{I F E} u-u_{h}^{J} & =u-I_{h}^{I F E} u-u^{J}+u^{J}-u_{h}^{J} \\
& =u-I_{h}^{I F E} u-\left(\widehat{u}-I_{h}^{I F E} \widehat{u}\right)+H(\varphi)\left(\widetilde{u}-I_{h}^{L} \widetilde{u}\right) \\
& =u-\widehat{u}-I_{h}^{I F E}(u-\widehat{u})+H(\varphi)\left(\widetilde{u}-I_{h}^{L} \widetilde{u}\right) \\
& =u^{h o m}-I_{h}^{I F E} u^{h o m}+H(\varphi)\left(\widetilde{u}-I_{h}^{L} \widetilde{u}\right) .
\end{aligned}
$$

Since $h<\rho$, one has $T \subset N(\Gamma, \rho)$, so that the assumption $\widetilde{u} \in H^{2}(N(\Gamma, \rho))$, relations (2.8) and Lemma 2.1 lead to the estimates

$$
\begin{aligned}
& \left\|u^{h o m}-I_{h}^{I F E} u^{h o m}\right\|_{L^{2}(T)} \leq C h^{2}\left(\|u\|_{H^{2}\left(T^{+} \cup T^{-}\right)}+\|\tilde{u}\|_{H^{2}\left(T^{+}\right)}\right), \\
& \left\|u^{h o m}-I_{h}^{I F E} u^{h o m}\right\|_{H^{1}(T)} \leq C h\left(\|u\|_{H^{2}\left(T^{+} \cup T^{-}\right)}+\|\tilde{u}\|_{H^{2}\left(T^{+}\right)}\right),
\end{aligned}
$$

with $T^{ \pm}:=T \cap \Omega^{ \pm}$. The third term in the right hand side of (2.14) can be estimated as follows:

$$
\left\|H(\varphi)\left(\widetilde{u}-I_{h}^{L} \widetilde{u}\right)\right\|_{L^{2}(T)}=\left\|\widetilde{u}-I_{h}^{L} \widetilde{u}\right\|_{L^{2}\left(T^{+}\right)} \leq\left\|\tilde{u}-I_{h}^{L} \widetilde{u}\right\|_{L^{2}(T)} \leq C h^{2}\|\widetilde{u}\|_{H^{2}(T)},
$$
and

$$
\left\|H(\varphi)\left(\widetilde{u}-I_{h}^{L} \widetilde{u}\right)\right\|_{H^{1}\left(T^{+} \cup T^{-}\right)}=\left\|\tilde{u}-I_{h}^{L} \widetilde{u}\right\|_{H^{1}\left(T^{+}\right)} \leq\left\|\tilde{u}-I_{h}^{L} \widetilde{u}\right\|_{H^{1}(T)} \leq C h\|\widetilde{u}\|_{H^{2}(T)} .
$$

For elements $T \in \mathscr{T}_{h}^{\text {non }} \backslash \mathscr{T}_{h}^{d}$, we have $u_{h}^{J}=0-$ cf. (2.11), hence

$$
\begin{aligned}
& \left\|u-I_{h}^{I F E} u-u_{h}^{J}\right\|_{L^{2}(T)}=\left\|u-I_{h}^{L} u\right\|_{L^{2}(T)} \leq C h^{2}\|u\|_{H^{2}(T)}, \\
& \left\|u-I_{h}^{I F E} u-u_{h}^{J}\right\|_{H^{1}(T)}=\left\|u-I_{h}^{L} u\right\|_{H^{1}(T)} \leq C h\|u\|_{H^{2}(T)},
\end{aligned}
$$

recalling that $I_{h}^{I F E}=I_{h}^{L}$ for non-interface elements. Putting together the relations (2.14)(2.18), we obtain inequalities (2.13), completing the proof of the lemma.

\subsection{A partially penalised IFE method}

Let $e$ be an edge shared by elements $T_{1}$ and $T_{2}$ and $\mathbf{n}_{i}$ be the unit normal to $e$ directed outside of $T_{i}, i=1,2$. The average $\{\{\nabla u\}\}$ and the jump $\llbracket u \rrbracket \mathbf{n}_{1}$ on the edge $e$ are defined 
by

$$
\begin{aligned}
& \{\nabla u\}\}:=\frac{1}{2}\left(\left.\nabla u\right|_{T_{1}}+\left.\nabla u\right|_{T_{2}}\right), \\
& \llbracket u \rrbracket \mathbf{n}_{1}:=\left.u\right|_{T_{1}} \mathbf{n}_{1}+\left.u\right|_{T_{2}} \mathbf{n}_{2}=\left(\left.u\right|_{T_{1}}-\left.u\right|_{T_{2}}\right) \mathbf{n}_{1} .
\end{aligned}
$$

For any edge $e \in \mathscr{E}_{h}$, we fix the unit normal vector and denote it by $\mathbf{n}_{e}$. For $e \subset \partial \Omega$, we define $\left\{\{v\}=\llbracket v \rrbracket=\left.v\right|_{T}\right.$.

The finite element method considered here consists in finding $u_{h}=u_{h}^{\text {hom }}+u_{h}^{J}$, $u_{h}^{\text {hom }} \in$ $V_{h}^{I F E}$ such that

$$
a_{h}\left(u_{h}^{h o m}, v_{h}\right)=\int_{\Omega} f v_{h} d x d y-\int_{\Gamma} Q v_{h} d s-a_{h}\left(u_{h}^{J}, v_{h}\right) \forall v_{h} \in V_{h}^{I F E},
$$

where $a_{h}(\cdot, \cdot)$ is the bilinear form

$$
\begin{aligned}
a_{h}\left(w, v_{h}\right)= & \sum_{T \in \mathscr{T}_{h}} \sum_{i= \pm} \int_{T \cap \Omega^{i}} \beta \nabla w \cdot \nabla v_{h} d x d y \\
& \left.-\sum_{e \in \mathcal{E}_{h}^{\text {int }}, e \not \subset} \int_{e}(\{\beta \nabla w\}\} \cdot \mathbf{n}_{e} \llbracket v_{h} \rrbracket+\left\{\left\{\beta \nabla v_{h}\right\}\right\} \cdot \mathbf{n}_{e} \llbracket u_{h}^{h o m} \rrbracket-\frac{\eta}{h} \llbracket w \rrbracket \llbracket v_{h} \rrbracket\right) d s,
\end{aligned}
$$

with a penalty parameter $\eta \geq 0$.

Remark 2.2. The function $u^{J}$ satisfies the non-homogeneous interface jump conditions, so the use of $u^{J}$ instead of $u_{h}^{J}$ looks more attractive. However, the implementation of such a method is more involved.

\subsection{A closest-point projection}

One of main difficulties in implementation of the method consists in the determination of the projections $\left(X^{*}, Y^{*}\right)$ of nodal points $\left(x_{i}, y_{i}\right) \in \mathscr{N}_{h}$ near the interface. For interfaces given implicitly by a signed distance function $d(x, y)$, one can use the Newton iterative method to find the root $\alpha$ of the one-dimensional nonlinear equation $d\left(x_{i}+\left.\alpha \frac{\partial d}{\partial x}\right|_{\left(x_{i}, y_{i}\right)}, y_{i}+\right.$ $\left.\left.\alpha \frac{\partial d}{\partial y}\right|_{\left(x_{i}, y_{i}\right)}\right)=0$ and obtain $\left(X^{*}, Y^{*}\right)=\left(x_{i}, y_{i}\right)+\alpha \nabla d\left(x_{i}, y_{i}\right)$.

In the case where the corresponding level set function $\varphi(x, y)$ is not a signed distance function, the projection $\left(X^{*}, Y^{*}\right)$ and the signed distance $d\left(x_{i}, y_{i}\right)$ can be simultaneously 
derived by the Newton iterative method for the coupled nonlinear equations - cf. Ref. [33]:

$$
\begin{aligned}
& X^{*}-x_{i}+\left.d\left(x_{i}, y_{i}\right) \frac{\frac{\partial \varphi}{\partial x}}{\sqrt{\left(\frac{\partial \varphi}{\partial x}\right)^{2}+\left(\frac{\partial \varphi}{\partial y}\right)^{2}}}\right|_{\left(X^{*}, Y^{*}\right)}=0 \\
& Y^{*}-y_{i}+\left.d\left(x_{i}, y_{i}\right) \frac{\frac{\partial \varphi}{\partial y}}{\sqrt{\left(\frac{\partial \varphi}{\partial x}\right)^{2}+\left(\frac{\partial \varphi}{\partial y}\right)^{2}}}\right|_{\left(X^{*}, Y^{*}\right)}=0 \\
& \varphi\left(X^{*}, Y^{*}\right)=0 .
\end{aligned}
$$

We note that the system (2.20) consists of three equations with three unknowns $X^{*}, Y^{*}$, $d\left(x_{i}, y_{i}\right)$. The initial value is chosen according to a method developed in Ref. [20, p. 76]. In our computations, the tolerance of the Newton iterative method was set as $10^{-12}$, and it takes only a few iterations to establish the closest-point projection.

For interfaces given by a parametrisation such as

$$
\left.\Gamma:=\left\{(x(t), y(t)): x, y \in C^{2}(I, \mathbb{R}), I \subset \mathbb{R}\right)\right\},
$$

the closest-point projection can be derived according to Ref. [33], so that our method is also applicable to problems with parametric representation of the interface.

\subsection{Problems with variable coefficients}

In this section, we show how to apply the partially penalised IFE method to the problem

$$
\begin{aligned}
& \beta(x, y)= \begin{cases}\beta^{-}(x, y), & (x, y) \in \Omega^{-}, \\
\beta^{+}(x, y), & (x, y) \in \Omega^{+},\end{cases} \\
& 0<\beta_{\min } \leq \beta(x, y) \leq \beta_{\max }<\infty
\end{aligned}
$$

with sufficiently smooth coefficients $\beta^{ \pm}(x, y)$. In order to work in an appropriate IFE space, the third equation in (2.3) is replaced by the equation

$$
\overline{\beta^{+}} \nabla v_{h}^{+} \cdot \mathbf{n}_{h}=\overline{\beta^{-}} \nabla v_{h}^{-} \cdot \mathbf{n}_{h},
$$

where $\overline{\beta^{ \pm}}$are the averages on the curve $\Gamma \cap \triangle A B C$, i.e.

$$
\overline{\beta^{ \pm}}=\frac{1}{|\Gamma \cap \triangle A B C|} \int_{\Gamma \cap \triangle A B C} \beta^{ \pm}(x, y) d s .
$$

We also will use a new correction function $u_{h}^{J}$. In case of variable coefficients, the equation (2.5) takes form

$$
\widetilde{u}(x, y)=w^{e}(x, y)+\frac{Q^{e}(x, y)}{\beta^{+, e}(x, y)} d(x, y), \quad(x, y) \in N(\Gamma, \rho),
$$

where

$$
\beta^{+, e}(x, y)=\beta^{+}(X, Y)
$$

with the closest-point projection $(X, Y)$ of $(x, y)$. 


\section{A Priori Error Estimates}

Introducing the space

$$
\begin{array}{r}
H_{h}:=\left\{v \in L^{2}(\Omega):\left.v\right|_{T^{ \pm}} \in H^{2}\left(T^{ \pm}\right),\left.v\right|_{T} \in H^{1}(T), \forall T \in \mathscr{T}_{h}, T^{ \pm}=T \cap \Omega^{ \pm},\right. \\
\text {and } \left.v \text { is continuous on } e \in \mathscr{E}_{h}^{\text {non }}\right\},
\end{array}
$$

we can consider a bilinear form $a_{h}(\cdot, \cdot)$ as acting on $H_{h} \times H_{h}$. For every $v \in H_{h}$, its energy norm is defined by

$$
\|v\|_{h}=\left(\sum_{T \in \mathscr{T}_{h}} \sum_{i= \pm} \int_{T \cap \Omega^{i}} \beta \nabla v \cdot \nabla v d x d y+\sum_{e \in \mathcal{E}_{h}^{\text {int }}, e \not \subset \Gamma} \int_{e} \frac{\eta}{h} \llbracket v \rrbracket \llbracket v \rrbracket d s\right)^{1 / 2} .
$$

The bilinear form $a_{h}(\cdot, \cdot)$ is coercive on the IFE space $V_{h}^{I F E}$ with respect to the energy norm $\|\cdot\|_{h}$ as is stated in the following lemma.

Lemma 3.1 (cf. Lin et al. [24]). There exists a constant $\kappa>0$, independent of $h$, such that for all sufficiently large penalty parameters $\eta$ and for all $v_{h} \in V_{h}^{I F E}$ the inequality

$$
a_{h}\left(v_{h}, v_{h}\right) \geq \kappa\left\|v_{h}\right\|_{h}^{2}
$$

holds.

Let us show the consistency of our method.

Lemma 3.2. If $u \in H^{2}\left(\Omega^{+} \cup \Omega^{-}\right)$is a solution of (1.1)-(1.4) and $u_{h}$ is a solution of (2.19), then for any $v_{h} \in V_{h}^{I F E}$ one has

$$
a_{h}\left(u-u_{h}, v_{h}\right)=0 .
$$

Proof. Let $T \in \mathscr{T}_{h}$ and $\mathscr{D}:=T \cap \Omega^{+}$or $\mathscr{D}:=T \cap \Omega^{-}$. Using integration by parts, for any $v_{h} \in V_{h}^{I F E}$ we obtain

$$
\begin{aligned}
\int_{\mathscr{D}} f v_{h} d x d y & =\int_{\mathscr{D}}-\nabla(\beta \nabla u) v_{h} d x d y \\
& =\int_{\mathscr{D}} \beta \nabla u \cdot \nabla v_{h} d x d y-\int_{\partial \mathscr{D}}\left(\beta \nabla u v_{h}\right) \cdot v d s,
\end{aligned}
$$

where $v$ is the outward unit normal to $\partial \mathscr{D}$. Summing up Eqs. (3.2) over all subdomains $\mathscr{D}$, 
we arrive at the representation

$$
\begin{aligned}
& \int_{\Omega} f v_{h} d x d y \\
= & \sum_{T \in \mathscr{T}_{h}} \sum_{i= \pm} \int_{T \cap \Omega^{i}} \beta \nabla u \cdot \nabla v_{h} d x d y-\sum_{e \in \mathscr{E}_{h}, e \subset \Gamma \Gamma} \int_{e} \llbracket \beta \nabla u v_{h} \rrbracket \cdot \mathbf{n}_{e} d s-\int_{\Gamma} \llbracket \beta \nabla u v_{h} \rrbracket \cdot \mathbf{n} d s \\
= & \sum_{T \in \mathscr{T}_{h}} \sum_{i= \pm} \int_{T \cap \Omega^{i}} \beta \nabla u \cdot \nabla v_{h} d x d y-\sum_{e \in \mathcal{E}_{h}, e \not \Gamma \Gamma} \int_{e}\left(\{\beta \nabla u\} \llbracket v_{h} \rrbracket+\left\{\left\{v_{h}\right\} \llbracket \beta \nabla u \rrbracket\right) \cdot \mathbf{n}_{e} d s\right. \\
& -\int_{\Gamma}\left(\left\{\{\beta \nabla u\} \llbracket v_{h} \rrbracket+\left\{\left\{v_{h}\right\}\right\} \llbracket \beta \nabla u \rrbracket\right) \cdot \mathbf{n} d s\right. \\
= & \sum_{T \in \mathscr{T}_{h}} \sum_{i= \pm} \int_{T \cap \Omega^{i}} \beta \nabla u \cdot \nabla v_{h} d x d y-\sum_{e \in \operatorname{E}_{h}^{\text {int }}, e \not \subset \Gamma} \int_{e}\left\{\{\beta \nabla u\} \llbracket v_{h} \rrbracket \cdot \mathbf{n}_{e} d s+\int_{\Gamma} Q v_{h} d s .\right.
\end{aligned}
$$

Note that in the above transformations, we used the relations:

1. $\llbracket a b \rrbracket=\{a a\} \llbracket b b \rrbracket+\{\{b\}\} \llbracket a \rrbracket ;$

2. $\llbracket v_{h} \rrbracket=0$ on $\Gamma$ and the jump condition (1.4);

3. $\llbracket v_{h} \rrbracket=0$ on $e \in \mathscr{E}_{h}^{\text {non }}$ and $\llbracket \beta \nabla u \cdot \mathbf{n}_{e} \rrbracket=0$ on $e \in \mathscr{E}_{h}, e \not \subset \Gamma$.

Moreover, since $u \in H^{2}\left(\Omega^{+} \cup \Omega^{-}\right)$, it implies $\llbracket u \rrbracket=0$ on $e \in \mathscr{E}_{h}^{\text {int }}, e \not \subset \Gamma$ and subsequently

$$
\sum_{e \in \mathscr{E}_{h}^{\text {int }}, e \not \subset \Gamma} \int_{e}\left\{\left\{\beta \nabla v_{h}\right\}\right\} \cdot \mathbf{n}_{e} \llbracket u \rrbracket d s=\sum_{e \in \mathcal{E}_{h}^{\text {int }}, \ell \subset \Gamma \Gamma} \int_{e} \frac{\eta}{h} \llbracket u \rrbracket \llbracket u \rrbracket d s=0 .
$$

It follows from (3.3), (3.4) that

$$
a_{h}\left(u, v_{h}\right)=\int_{\Omega} f v_{h} d x d y-\int_{\Gamma} Q v_{h} d s \text { for all } v_{h} \in V_{h}^{I F E} .
$$

Combining it with Eq. (2.19), we obtain

$$
a_{h}\left(u-u_{h}, v_{h}\right)=0 \text { for all } \in V_{h}^{I F E},
$$

which completes the proof.

In order to derive error estimates in energy norm, we need a result from Ref. [24].

Lemma 3.3 (cf. Lin et al. [24]). Let $\Omega_{\rho}^{ \pm}:=\Omega^{ \pm} \cap N(\Gamma, \rho)$. If $h<\rho$, then for any $v \in$ $H^{3}\left(\Omega_{\rho}^{+} \cup \Omega_{\rho}^{-}\right)$satisfying the conditions $[v]_{\Gamma}=0$ and $\left[\beta \frac{\partial v}{\partial \mathbf{n}}\right]_{\Gamma}=0$, there is a constant $C$, independent of $\Gamma$, such that

$$
\left\|\left.\left(\beta \nabla\left(v-I_{h}^{I F E} v\right)\right)\right|_{T} \cdot \mathbf{n}_{e}\right\|_{L^{2}\left(e^{+} \cup e^{-}\right)}^{2} \leq C\left(h^{2}\|v\|_{H^{3}\left(\Omega_{\rho}^{+} \cup \Omega_{\rho}^{-}\right)}^{2}+h\|v\|_{H^{2}\left(T^{+} \cup T^{-}\right)}^{2}\right),
$$

where $I_{h}^{I F E} v$ is the interpolation of $v$ in $V_{h}^{I F E}, e^{ \pm}=e \cap \Omega^{ \pm}, T^{ \pm}=T \cap \Omega^{ \pm}$, and $e$ is an edge of the interface element $T$. 
This lemma allows us to establish the following result.

Corollary 3.1. If the function $\widetilde{u}$ of (2.9) belongs to $H^{3}(N(\Gamma, \rho))$, then for any $u \in H^{3}\left(\Omega^{+} \cup\right.$ $\left.\Omega^{-}\right)$satisfying the non-homogeneous interface conditions (1.3)-(1.4), there is a constant $C$, independent of $\Gamma$, such that for any $h<\rho$ the estimate

$$
\begin{aligned}
& \left\|\left.\left(\beta \nabla\left(u-I_{h}^{I F E} u-u_{h}^{J}\right)\right)\right|_{T} \cdot \mathbf{n}_{e}\right\|_{L^{2}\left(e^{+} \cup e^{-}\right)}^{2} \\
\leq & C\left(h^{2}\left(\|u\|_{H^{3}\left(\Omega_{\rho}^{+} \cup \Omega_{\rho}^{-}\right)}^{2}+\|\widetilde{u}\|_{H^{3}\left(\Omega_{\rho}^{+}\right)}^{2}\right)+h\left(\|u\|_{H^{2}\left(T^{+} \cup T^{-}\right)}^{2}+\|\widetilde{u}\|_{H^{2}(T)}^{2}\right)\right),
\end{aligned}
$$

holds.

Proof. According to (2.7), we have $u^{\text {hom }}(x, y)=u(x, y)-\widehat{u}(x, y)$ in $N(\Gamma, \rho)$, so that for an interface element $T \in \mathscr{T}_{h}^{\text {int }}$, the relation (2.14) yields

$$
u-I_{h}^{I F E} u-u_{h}^{J}=\left(u^{h o m}-I_{h}^{I F E} u^{h o m}\right)+H(\varphi)\left(\widetilde{u}-I_{h}^{L} \widetilde{u}\right) .
$$

Since $u^{\text {hom }}$ satisfies the conditions of Lemma 3.3, we have

$$
\begin{aligned}
& \left\|\left.\left(\beta \nabla\left(u^{h o m}-I_{h}^{I F E} u^{h o m}\right)\right)\right|_{T} \cdot \mathbf{n}_{e}\right\|_{L^{2}(e)}^{2} \\
\leq & C\left(h^{2}\left\|u^{h o m}\right\|_{H^{3}\left(\Omega_{\rho}^{+} \cup \Omega_{\rho}^{-}\right)}^{2}+h\left\|u^{h o m}\right\|_{H^{2}\left(T^{+} \cup T^{-}\right)}^{2}\right) \\
\leq & C\left(h^{2}\left(\|u\|_{H^{3}\left(\Omega_{\rho}^{+} \cup \Omega_{\rho}^{-}\right)}^{2}+\|\widetilde{u}\|_{H^{3}\left(\Omega_{\rho}^{+}\right)}^{2}\right)+h\left(\|u\|_{H^{2}\left(T^{+} \cup T^{-}\right)}^{2}+\|\widetilde{u}\|_{H^{2}\left(T^{+}\right)}^{2}\right)\right) .
\end{aligned}
$$

The second term in the right-hand side of (3.5) can be estimated by using the standard trace inequality, so that

$$
\begin{aligned}
& \|\left(\beta \nabla ( H ( \varphi ) ( \widetilde { u } - I _ { h } ^ { L } \widetilde { u } ) ) | _ { T } \cdot \mathbf { n } _ { e } \| _ { L ^ { 2 } ( e ^ { + } \cup e ^ { - } ) } ^ { 2 } = \| \left(\left.\beta \nabla\left(\widetilde{u}-I_{h}^{L} \widetilde{u}\right)\right|_{T} \cdot \mathbf{n}_{e} \|_{L^{2}\left(e^{+}\right)}^{2}\right.\right. \\
\leq & \|\left(\left.\beta \nabla\left(\widetilde{u}-I_{h}^{L} \widetilde{u}\right)\right|_{T} \cdot \mathbf{n}_{e} \|_{L^{2}(e)}^{2} \leq C\left(h^{-1}\left|\widetilde{u}-I_{h}^{L} \widetilde{u}\right|_{H^{1}(T)}^{2}+h\left|\widetilde{u}-I_{h}^{L} \widetilde{u}\right|_{H^{2}(T)}^{2}\right)\right. \\
\leq & C h|\widetilde{u}|_{H^{2}(T)}^{2},
\end{aligned}
$$

and the result follows from (3.5)-(3.7).

Lemma 3.4. Assume that $h<\rho$ and the function $\widetilde{u}$ of (2.9) belongs to the space $H^{2}(N(\Gamma, \rho))$. Then

$$
\begin{aligned}
& \left\|\left.\left(u-I_{h}^{I F E} u-u_{h}^{J}\right)\right|_{T}\right\|_{L^{2}(e)}^{2} \\
\leq & C h^{-1}\left\|u-I_{h}^{I F E} u-u_{h}^{J}\right\|_{L^{2}(T)}^{2}+C h\left|u-I_{h}^{I F E} u-u_{h}^{J}\right|_{H^{1}(T)}^{2}+C h^{3}\|\widetilde{u}\|_{H^{2}(T)}^{2},
\end{aligned}
$$

where $T$ is an interface element and $e$ is an interface edge of $T$.

Proof. First of all, we write

$$
\left\|\left.\left(u-I_{h}^{I F E} u-u_{h}^{J}\right)\right|_{T}\right\|_{L^{2}(e)}^{2} \leq\left\|\left.\left(u-I_{h}^{I F E} u-u^{J}\right)\right|_{T}\right\|_{L^{2}(e)}^{2}+\left\|\left.\left(u^{J}-u_{h}^{J}\right)\right|_{T}\right\|_{L^{2}(e)}^{2} .
$$


Evaluating the first term in the right-hand side of (3.9), we note that $u-u^{J} \in H^{1}(T)$, $I_{h}^{I F E} u \in H^{1}(T)$ and apply the standard trace inequality. The second term can be estimated by using (2.11) and (2.12) and the standard trace inequality

$$
\begin{aligned}
\left\|\left.\left(u^{J}-u_{h}^{J}\right)\right|_{T}\right\|_{L^{2}(e)}^{2} & =\left\|H(\varphi)\left(\widetilde{u}-I_{h}^{L} \widetilde{u}\right)\right\|_{L^{2}(e)}^{2} \leq\left\|\widetilde{u}-I_{h}^{L} \widetilde{u}\right\|_{L^{2}(e)}^{2} \\
& \leq C h^{-1}\left\|\widetilde{u}-I_{h}^{L} \widetilde{u}\right\|_{L^{2}(T)}^{2}+C h\left\|\widetilde{u}-I_{h}^{L} \widetilde{u}\right\|_{H^{1}(T)}^{2} \\
& \leq C h^{3}\|\widetilde{u}\|_{H^{2}(T)}^{2},
\end{aligned}
$$

hence inequality (3.8) follows.

Now we can establish error estimates for the proposed method.

Theorem 3.1. Assume that $h<\rho$, the function $\tilde{u}$ of (2.9) belongs to $H^{3}(N(\Gamma, \rho))$ and the exact solution $u$ of the interface problem (1.1)-(1.4) belongs to $H^{3}\left(\Omega^{+} \cup \Omega^{-}\right)$. If $u_{h}$ is the approximate solution obtained by the method (2.19), then

$$
\left\|u-u_{h}\right\|_{h} \leq C h\left(\|u\|_{H^{3}\left(\Omega^{+} \cup \Omega^{-}\right)}+\|\widetilde{u}\|_{H^{3}(N(\Gamma, \rho))}\right),
$$

with a constant $C$.

Proof. Since $u_{h}=u_{h}^{\text {hom }}+u_{h}^{J}$, we can write

$$
\left\|u-u_{h}\right\|_{h} \leq\left\|u-I_{h}^{I F E} u-u_{h}^{J}\right\|_{h}+\left\|I_{h}^{I F E} u-u_{h}^{h o m}\right\|_{h} .
$$

By Lemma 3.2,

$$
a_{h}\left(I_{h}^{I F E} u-u_{h}^{h o m}, v_{h}\right)=-a_{h}\left(u-I_{h}^{I F E} u-u_{h}^{J}, v_{h}\right), \quad v_{h} \in V_{h}^{I F E},
$$

so letting $v_{h}=I_{h}^{I F E} u-u_{h}^{\text {hom }}$ and using Lemma 3.1, one obtains

$$
\begin{aligned}
\kappa\left\|I_{h}^{I F E} u-u_{h}^{h o m}\right\|_{h}^{2} & \leq a_{h}\left(I_{h}^{I F E} u-u_{h}^{\text {hom }}, I_{h}^{I F E} u-u_{h}^{h o m}\right) \\
& \leq\left|a_{h}\left(u-I_{h}^{I F E} u-u_{h}^{J}, I_{h}^{I F E} u-u_{h}^{h o m}\right)\right| \\
& \leq\left|\sum_{T \in \mathscr{T}_{h}} \sum_{i= \pm} \int_{T \cap \Omega^{i}} \beta \nabla\left(u-I_{h}^{I F E} u-u_{h}^{J}\right) \cdot \nabla\left(I_{h}^{I F E} u-u_{h}^{\text {hom }}\right) d x d y\right| \\
& +\left|\sum_{e \in \mathscr{E}_{h}^{\text {int }}, e \not \subset \Gamma} \sum_{i= \pm} \int_{e \cap \Omega^{i}}\left\{\left\{\beta \nabla\left(u-I_{h}^{I F E} u-u_{h}^{J}\right)\right\}\right\} \cdot \mathbf{n}_{e} \llbracket I_{h}^{I F E} u-u_{h}^{h o m} \rrbracket d s\right| \\
& +\left|\sum_{e \in \mathscr{E}_{h}^{\text {int }}, e \not \subset \Gamma} \sum_{i= \pm} \int_{e \cap \Omega^{i}}\left\{\left\{\beta \nabla\left(I_{h}^{I F E} u-u_{h}^{h o m}\right)\right\}\right\} \cdot \mathbf{n}_{e} \llbracket u-I_{h}^{I F E} u-u_{h}^{J} \rrbracket d s\right| \\
& +\left|\sum_{e \in \mathscr{E}_{h}^{\text {int }}, e \not \subset \Gamma} \sum_{i= \pm} \int_{e \cap \Omega^{i}} \frac{\eta}{h} \llbracket u-I_{h}^{I F E} u-u_{h}^{J} \rrbracket \cdot \mathbf{n}_{e} \llbracket I_{h}^{I F E} u-u_{h}^{h o m} \rrbracket d s\right| .
\end{aligned}
$$


Next steps are similar to the proof of Theorem 4.3 in Ref. [24], and the use of Lemma 3.4 leads to the estimate

$$
\begin{aligned}
\left\|I_{h}^{I F E} u-u_{h}^{h o m}\right\|_{h}^{2} \leq & C\left|u-I_{h}^{I F E} u-u_{h}^{J}\right|_{H^{1}\left(\Omega^{+} \cup \Omega^{-}\right)}^{2}+C h^{-2}\left\|u-I_{h}^{I F E} u-u_{h}^{J}\right\|_{L^{2}\left(\Omega^{+} \cup \Omega^{-}\right)}^{2} \\
& +C \sum_{e \in \mathscr{E}_{h}^{\text {int }}, e \not \subset \Gamma}\left(h\left\|\left\{\left\{\beta \nabla\left(u-I_{h}^{I F E} u-u_{h}^{J}\right)\right\}\right\} \cdot \mathbf{n}_{e}\right\|_{L^{2}\left(e^{+} \cup e^{-}\right)}^{2}+h^{2}\|\tilde{u}\|_{H^{2}(N(\Gamma, \rho))}^{2}\right) .
\end{aligned}
$$

Taking into account Lemma 2.2, Corollary 3.1 and the fact that the number of the interface elements is $O\left(h^{-1}\right)$, we finally obtain

$$
\left\|I_{h}^{I F E} u-u_{h}^{h o m}\right\|_{h} \leq C h\left(\|u\|_{H^{3}\left(\Omega^{+} \cup \Omega^{-}\right)}+\|\widetilde{u}\|_{H^{3}(N(\Gamma, \rho))}\right) .
$$

The first term in the right-hand of (3.11) can be estimated analogously, so that

$$
\left\|u-I_{h}^{I F E} u-u_{h}^{J}\right\|_{h} \leq C h\left(\|u\|_{H^{2}\left(\Omega^{+} \cup \Omega^{-}\right)}+\|\widetilde{u}\|_{H^{2}(N(\Gamma, \rho))}\right) .
$$

The inequality (3.10) now follows on putting together estimates (3.11), (3.12) and (3.13).

\section{Numerical Examples}

Let us discuss a few numerical examples to show the efficiency of the method. For simplicity, we only consider the problem (1.1)-(1.4) in the rectangular domain $\Omega=[-1,1] \times$ $[-1,1]$ with known analytic solution $u^{+}(x, y), u^{-}(x, y)$, the coefficients $\beta^{+}(x, y), \beta^{-}(x, y)$ and the level set function $\varphi(x, y)$. The domain is partitioned into $2 N^{2}$ right triangles with the mesh size $h$. The integrals on interface elements are evaluated by splitting these elements into small triangles and using the three-point Gaussian quadrature. For non-interface elements, the three-point Gaussian quadrature is used directly. If $\varphi\left(x_{i}, y_{i}\right)$ is smaller than machine precision, it is not possible to decide whether a grid point $\left(x_{i}, y_{i}\right)$ belongs to $\Omega^{+}$. To avoid the problem, we set $\varphi\left(x_{i}, y_{i}\right)=0$ for $\left|\varphi\left(x_{i}, y_{i}\right)\right|<10^{-13}$. In all numerical examples, the penalty parameter $\eta$ is set to 0 . In problems with variable coefficients, the averages $\overline{\beta^{ \pm}}$ defined in (2.21) are computed by the formula

$$
\overline{\beta^{ \pm}} \approx \frac{\beta^{ \pm}\left(x_{D}, y_{D}\right)+\beta^{ \pm}\left(x_{E}, y_{E}\right)}{2} .
$$

and this approximation does not influence the convergence order. The errors are measured in the broken $H^{1}(\Omega)$ norm, $L^{2}(\Omega)$ norm and in $L^{\infty}$ norm. The last one is calculated as maximum error at the grid points.

Example 4.1 (cf. Hou \& Liu [12]). The coefficients $\beta^{ \pm}$, the level set function $\varphi$ and the analytic solution $u^{ \pm}$of the interface problem are

$$
\begin{aligned}
& \beta^{+}=\sin (x+y)+2, \quad \beta^{-}=\cos (x+y)+2, \\
& \varphi=x^{2}+y^{2}-0.5^{2}-\epsilon, \\
& u^{+}=\ln \left(x^{2}+y^{2}\right), \quad u^{-}=\sin (x+y) .
\end{aligned}
$$


Table 1: Example 4.1. Grid refinement analysis.

\begin{tabular}{ccccccc}
\hline$N$ & $L^{2}$ error & Order & $H^{1}$ error & Order & $L^{\infty}$ error & Order \\
\hline 64 & $1.2815 \mathrm{E}-03$ & & $9.8904 \mathrm{E}-02$ & & $1.3084 \mathrm{E}-03$ & \\
128 & $3.2863 \mathrm{E}-04$ & 1.96 & $4.9569 \mathrm{E}-02$ & 0.99 & $3.3643 \mathrm{E}-04$ & 1.95 \\
256 & $8.2955 \mathrm{E}-05$ & 1.98 & $2.4815 \mathrm{E}-02$ & 0.99 & $8.5487 \mathrm{E}-05$ & 1.97 \\
512 & $2.0798 \mathrm{E}-05$ & 1.99 & $1.2418 \mathrm{E}-02$ & 0.99 & $2.1318 \mathrm{E}-05$ & 2.00 \\
1024 & $5.2188 \mathrm{E}-06$ & 1.99 & $6.2112 \mathrm{E}-03$ & 0.99 & $5.3312 \mathrm{E}-06$ & 1.99 \\
\hline
\end{tabular}
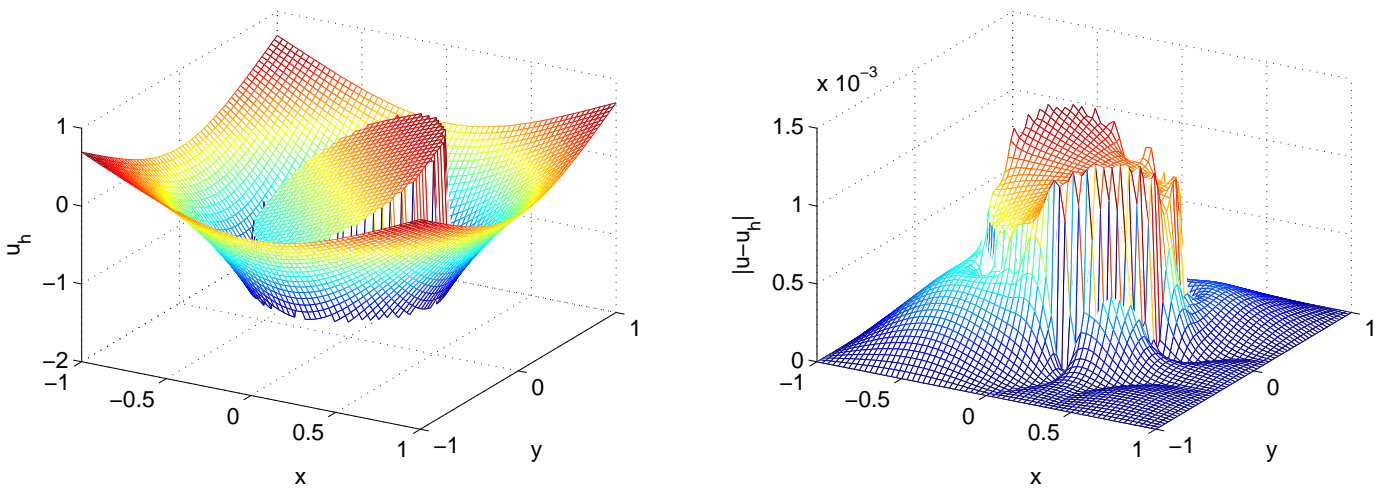

Figure 4: Example 4.1. Left: Approximate solution, $N=64$. Right: Error distribution.

Let us start with the case $\epsilon=0$. The interface is a circle of radius 0.5 with the center $(0,0)$. The interface contains grid points, e.g. $(0,0.5)$ and $(0.3,0.4)$. The results are presented in Table 1. We note that the method has optimal convergence in $L^{2}, H^{1}$ and $L^{\infty}$ norm. For $\mathrm{N}=64$, the corresponding approximate solution and the error distribution are shown in Fig. 4.

Let us now consider the case $\epsilon=10^{-12}$. The interface is a circle of radius $0.5+\sqrt{\epsilon}$. If $\left|\varphi\left(x_{i}, y_{i}\right)\right|<10^{-13}$, then we set $\varphi\left(x_{i}, y_{i}\right)=0$. In this situation, there are grid points $\left(x_{i}, y_{i}\right)$ - e.g. $(0,0.5),(0.3,0.4)$, such that $10^{-12}>\left|\varphi\left(x_{i}, y_{i}\right)\right|>10^{-13}$. Therefore, the interface splits certain interface elements in such a way that the ratio between the areas on two neighbouring regions becomes very large or very small. However, numerical results are exactly the same as for $\epsilon=0-$ cf. Table 1 .

Example 4.2 (cf. Li [19]). The coefficients $\beta^{ \pm}$are positive constants, and the level set function $\varphi$ and the analytic solution $u^{ \pm}$of the interface problem are

$$
\begin{aligned}
& \varphi=\sqrt{\left(x-x_{c}\right)^{2}+\left(y-y_{c}\right)^{2}}-\left(r_{0}+r_{1} \sin (\omega \theta)\right), \\
& u^{+}=\frac{r^{4}-C_{0} \log (2 r)}{\beta^{+}}, \quad u^{-}=\frac{r^{2}}{\beta^{-}},
\end{aligned}
$$

where $(r, \theta)$ are the polar coordinates of $(x, y), C_{0}=-0.1,\left(x_{c}, y_{c}\right)=(0.2 / \sqrt{20}, 0.2 / \sqrt{20})$, $r_{0}=0.5, r_{1}=0.2$, and $\omega=5$. 
Table 2: Example 4.2. Grid refinement analysis, $\beta^{+}=10, \beta^{-}=1$.

\begin{tabular}{ccccccc}
\hline$N$ & $L^{2}$ error & Order & $H^{1}$ error & Order & $L^{\infty}$ error & Order \\
\hline 64 & $4.8922 \mathrm{E}-04$ & & $4.9366 \mathrm{E}-02$ & & $6.1616 \mathrm{E}-03$ & \\
128 & $9.4417 \mathrm{E}-05$ & 2.37 & $1.8894 \mathrm{E}-02$ & 1.38 & $4.8933 \mathrm{E}-04$ & 3.65 \\
256 & $2.3007 \mathrm{E}-05$ & 2.03 & $8.6065 \mathrm{E}-03$ & 1.13 & $2.9316 \mathrm{E}-04$ & 0.73 \\
512 & $5.6912 \mathrm{E}-06$ & 2.01 & $4.0630 \mathrm{E}-03$ & 1.08 & $6.0976 \mathrm{E}-05$ & 2.26 \\
1024 & $1.4531 \mathrm{E}-07$ & 1.96 & $1.9660 \mathrm{E}-03$ & 1.04 & $1.3368 \mathrm{E}-05$ & 2.18 \\
\hline
\end{tabular}
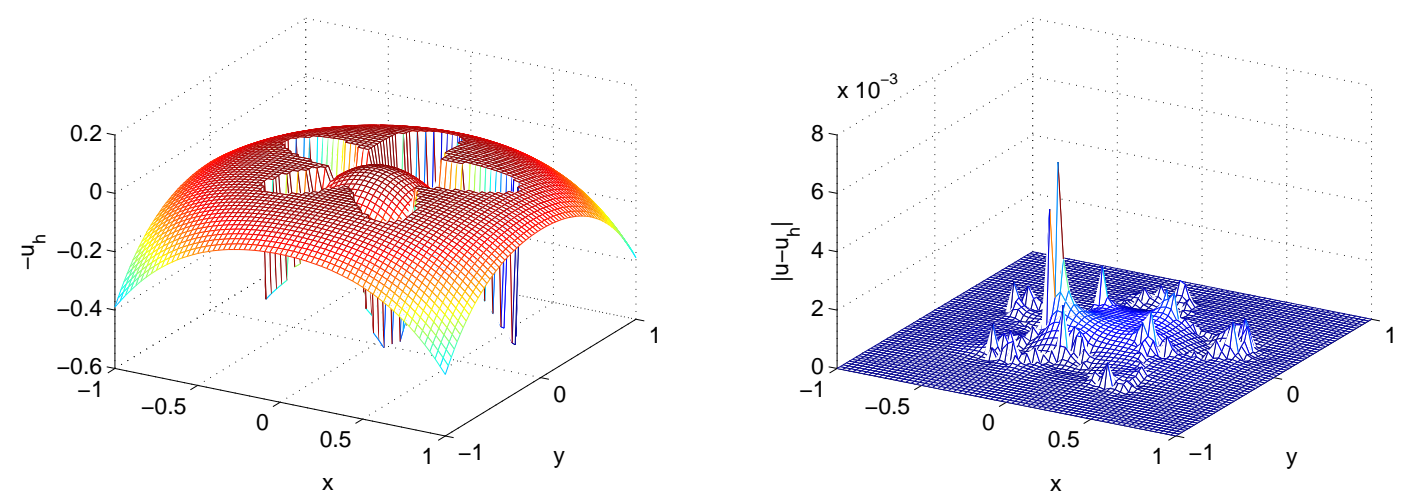

Figure 5: Example 4.2. Left: Approximate solution $-u_{h} ; \beta^{+}=10, \beta^{-}=1, N=64$. Right: Error distribution.
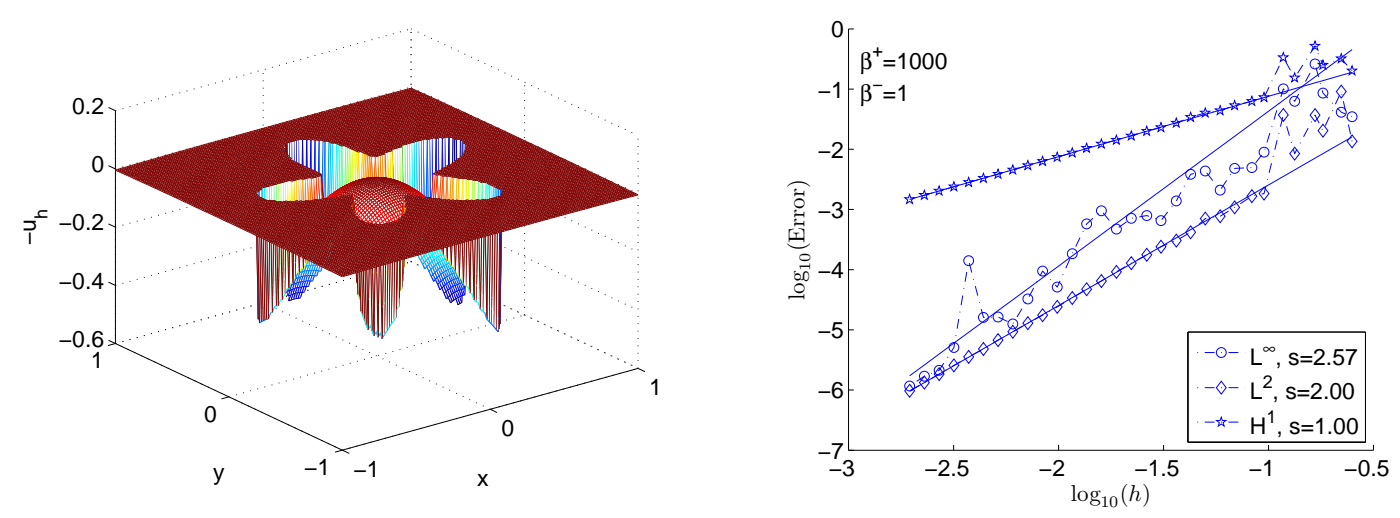

Figure 6: Example 4.2. Left: Approximate solution $-u_{h} ; \beta^{+}=1000, \beta^{-}=1, N=128$. Right: Linear regression analysis for three norms in log-log scale.

The interface in this example is more involved. In Table 2 and Figs. 5-7 we report numerical results for three typical cases - viz. for a moderate jump: $\beta^{+}=10, \beta^{-}=1$ and for two large jumps: $\beta^{+}=1000, \beta^{-}=1$ and $\beta^{+}=1, \beta^{-}=1000$.

Example 4.3 (cf. Li \& Ito [20, Example 3.2, p. 50]). The coefficients $\beta^{ \pm}$, the level set 

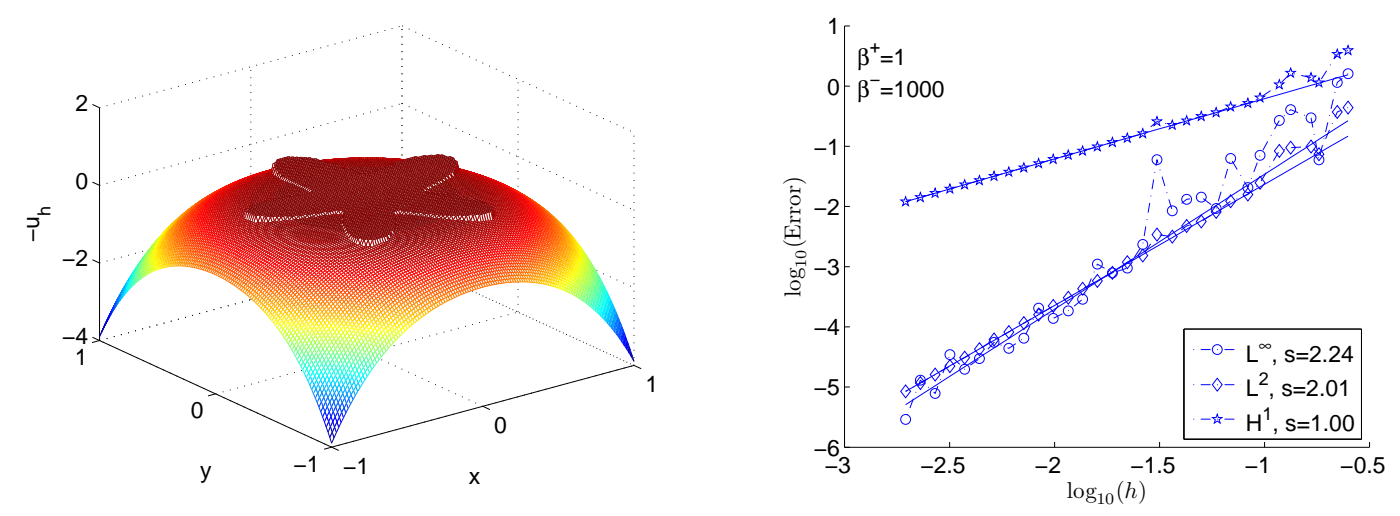

Figure 7: Example 4.2. Left: Approximate solution $-u_{h} ; \beta^{+}=1, \beta^{-}=1000, N=128$. Right: Linear regression analysis for three norms in log-log scale.

Table 3: Example 4.3. Grid refinement analysis.

\begin{tabular}{ccccccc}
\hline$N$ & $L^{2}$ error & Order & $H^{1}$ error & Order & $L^{\infty}$ error & Order \\
\hline 64 & $1.1078 \mathrm{E}-03$ & & $1.1098 \mathrm{E}-01$ & & $2.4818 \mathrm{E}-03$ & \\
128 & $2.7858 \mathrm{E}-04$ & 1.99 & $5.4595 \mathrm{E}-02$ & 1.02 & $8.1044 \mathrm{E}-04$ & 1.61 \\
256 & $7.0648 \mathrm{E}-05$ & 1.97 & $2.7037 \mathrm{E}-02$ & 1.01 & $2.5290 \mathrm{E}-04$ & 1.68 \\
512 & $1.7641 \mathrm{E}-05$ & 2.00 & $1.3402 \mathrm{E}-02$ & 1.01 & $6.4026 \mathrm{E}-05$ & 1.98 \\
1024 & $4.4561 \mathrm{E}-06$ & 1.98 & $6.6759 \mathrm{E}-03$ & 1.00 & $1.6532 \mathrm{E}-05$ & 1.95 \\
\hline
\end{tabular}

function $\varphi$ and the analytic solution $u^{ \pm}$of the interface problem are

$$
\begin{aligned}
& \beta^{+}=10, \quad \beta^{-}=1, \\
& \varphi=x^{2}+(2 y)^{2}-0.5^{2}, \\
& u^{+}=\sin (2 x) \cos (2 y), \quad u^{-}=(2 x)^{2}-(2 y)^{2} .
\end{aligned}
$$

The results are presented in Table 3 and Fig. 8.

Example 4.4. The coefficients $\beta^{ \pm}$, the level set function $\varphi$ and the analytic solution $u^{ \pm}$of the interface problem are

$$
\begin{aligned}
& \beta^{+}=10, \quad \beta^{-}=1 \\
& \varphi=\left(3\left(x^{2}+y^{2}\right)-x\right)^{2}-x^{2}-y^{2}+0.02 \\
& u^{+}=\frac{1}{2} \cos \left(1-x^{2}-y^{2}\right), \quad u^{-}=\sin \left(2 x^{2}+y^{2}+2\right) .
\end{aligned}
$$

The interface is heart shaped and the results are presented in Table 4 and Fig. 9. 

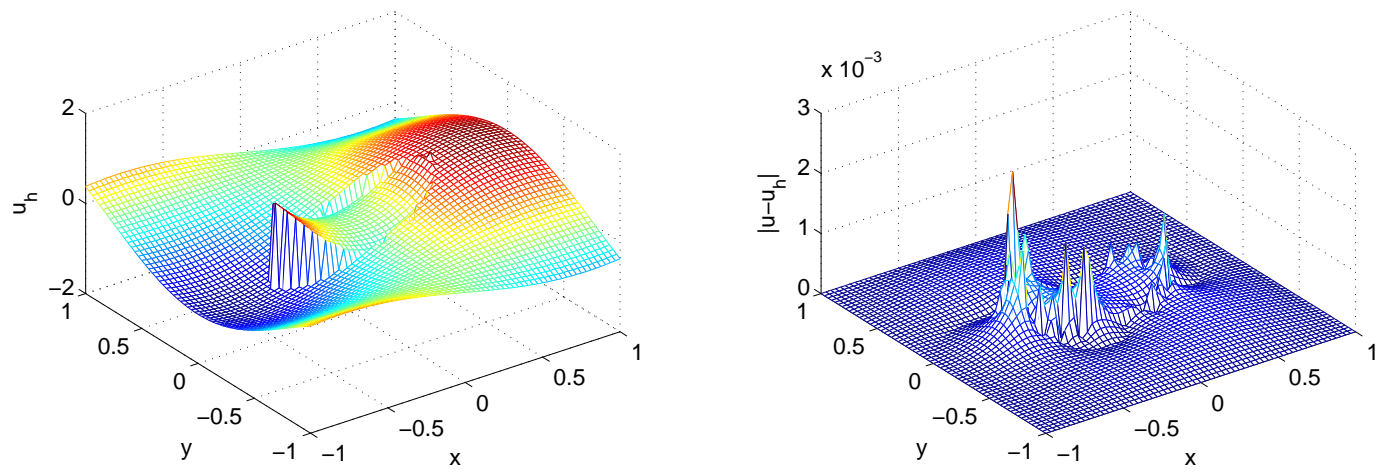

Figure 8: Example 4.3. Left: Approximate solution $u_{h} ; N=64$. Right: error distribution.

Table 4: Example 4.4. Grid refinement analysis.

\begin{tabular}{ccccccc}
\hline$N$ & $L^{2}$ error & Order & $H^{1}$ error & Order & $L^{\infty}$ error & Order \\
\hline 64 & $4.0089 \mathrm{E}-04$ & & $4.3745 \mathrm{E}-02$ & & $1.1634 \mathrm{E}-03$ & \\
128 & $9.9975 \mathrm{E}-05$ & 2.00 & $2.1382 \mathrm{E}-02$ & 1.03 & $5.3075 \mathrm{E}-04$ & 1.13 \\
256 & $2.5062 \mathrm{E}-05$ & 1.99 & $1.0507 \mathrm{E}-02$ & 1.02 & $1.3197 \mathrm{E}-04$ & 2.00 \\
512 & $6.2350 \mathrm{E}-06$ & 2.00 & $5.2154 \mathrm{E}-03$ & 1.01 & $3.9363 \mathrm{E}-05$ & 1.74 \\
1024 & $1.5190 \mathrm{E}-06$ & 2.03 & $2.5970 \mathrm{E}-03$ & 1.00 & $1.0011 \mathrm{E}-05$ & 1.97 \\
\hline
\end{tabular}
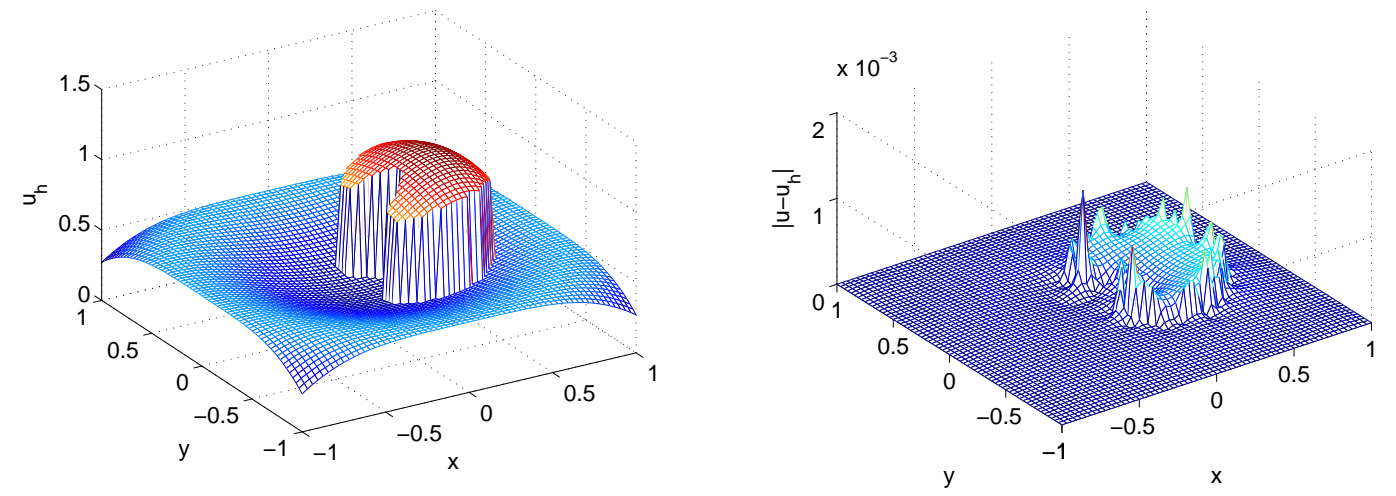

Figure 9: Example 4.4. Left: Approximate solution $u_{h} ; N=64$. Right: Error distribution.

Example 4.5 (cf. Hou \& Liu [12]). The coefficients $\beta^{ \pm}$, the level set function $\varphi$ and the analytic solution $u^{ \pm}$of the interface problem are

$$
\begin{aligned}
& \beta^{+}=x y+2, \quad \beta^{-}=x^{2}-y^{2}+3, \\
& \varphi=x^{2}-y-1, \\
& u^{+}=4-x^{2}-y^{2}, \quad u^{-}=x^{2}+y^{2} .
\end{aligned}
$$



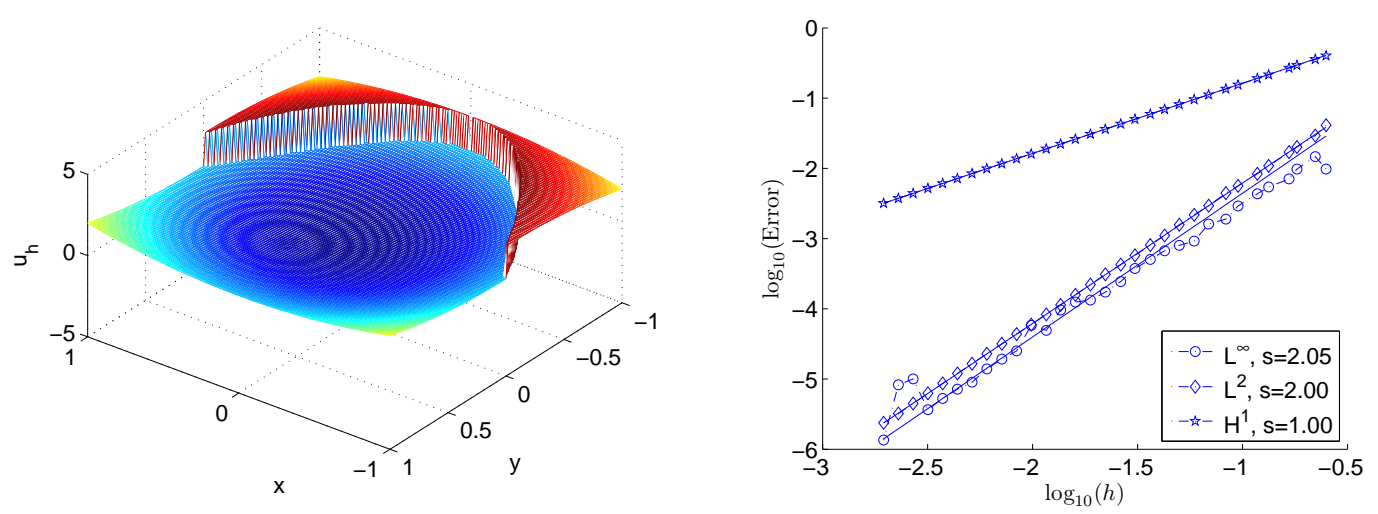

Figure 10: Example 4.5. Left: Approximate solution $u_{h} ; N=128$. Right: Linear regression analysis for three norms in log-log scale.

The interface is tangential to the boundary $\partial \Omega$ at the $(0,-1)$, and crosses it at the points $(-1,0),(1,0)$ under non-zero angles. Numerical results presented in Fig. 10 again demonstrate an optimal convergence of the method.

\section{Conclusion}

In this paper, we propose a partially penalised IFE method for interface problems with discontinuous coefficients and non-homogeneous jump conditions. The method is based on unfitted meshes independent of the interface. The degree of freedom is similar to that of traditional linear conforming finite element methods. The corresponding systems of linear equations have symmetric positive definite matrices which make possible the use of fast solvers. The implementation of the method is simple since slightly modified existing IFE codes can be used.

\section{Acknowledgments}

This work was supported in part by the Natural Science Foundation of the Jiangsu Higher Education Institution of China (Grant No. 17KJB110014), the Jiangsu Key Laboratory for NSLSCS (Grant Nos. 201704 and 201604), the National Science Foundation of China (Grant No. 11701291), the Natural Science Foundation of Jiangsu Province (Grant No. BK20160880), the China Postdoctoral Science Foundation (No. 2017T100354), and by NUPTSF (Grant Nos. NY216030 and NY217090).

\section{References}

[1] N. An and H. Chen, A partially penalty immersed interface finite element method for anisotropic elliptic interface problems, Numer. Methods Partial Differential Equations 30, 1984-2028 (2014). 
[2] J. Bedrossian, J. Brecht, S. Zhu, E. Sifakis and J. Teran, A second order virtual node method for elliptic problems with interface and irregular domains, J. Comput. Phys. 229, 6405-6426 (2010).

[3] K. Chang and D. Kwak, Discontinuous bubble scheme for elliptic problems with jumps in the solution, Comput. Methods Appl. Mech. Engrg. 200, 494-508 (2011).

[4] I. Chern and Y. Shu, A coupling interface method for elliptic interface problems, J. Comput. Phys. 225, 2138-2174 (2007).

[5] M. Discacciati, A. Quarteroni and S. Quinodoz, Numerical approximation of internal discontinuity interface problems, SIAM J. Sci. Comput. 35, A2341-A2369 (2013).

[6] T. Fries and T. Belytschko, The extended/generalized finite element method: An overview of the method and its applications, Int. J. Numer. Meth. Engng. 84 253-304 (2010).

[7] Y. Gong, B. Li and Z. Li, Immersed-interface finite-element methods for elliptic interface problems with non-homogeneous jump conditions, SIAM J. Numer. Anal. 46, 472-495 (2008).

[8] A. Hansbo and P. Hansbo, An unfitted finite element method, based on Nitsche's method, for elliptic interface problems, Comput. Methods Appl. Mech. Engrg. 191, 5537-5552 (2002).

[9] X. He, T. Lin and Y. Lin, Immersed finite element methods for elliptic interface problems with non-homogeneous jump conditions, Int. J. Numer. Anal. Model. 8, 284-301 (2011).

[10] X. He, T. Lin and Y. Lin, The convergence of the bilinear and linear immersed finite element solutions to interface problems, Numer. Methods Partial Differential Equations 28, 312-330 (2012).

[11] J. Hellrung, L. Wang, E. Sifakis and J. Teran, A second order virtual node method for elliptic problems with interfaces and irregular domains in three dimensions, J. Comput. Phys. 231, 2015-2048 (2012).

[12] S. Hou and X. Liu, A numerical method for solving variable coefficient elliptic equation with interfaces, J. Comput. Phys. 202, 411-445 (2005).

[13] S. Hou, P. Song, L. Wang and H. Zhao, A weak formulation for solving elliptic interface problems without body fitted grid, J. Comput. Phys. 249, 80-95 (2013).

[14] J. Huang and J. Zou, Uniform a priori estimates for elliptic and static Maxwell interface problems, Discrete and Continuous Dynamical Systems Series B 7, 145-170 (2007).

[15] H. Ji, J. Chen and Z. Li, A symmetric and consistent immersed finite element method for interface problems, J. Sci. Comput. 61, 533-557 (2014).

[16] H. Ji, J. Chen and Z. Li, Augmented immersed finite element methods for some elliptic partial differential equations, Int. J. Comput. Math. 93, 540-558 (2016).

[17] H. Ji, J. Chen and Z. Li, A new augmented immersed finite element method without using SVD interpolations, Numer. Algorithms 71, 395-416 (2016).

[18] R. LeVeque and Z. Li, The immersed interface method for elliptic equations with discontinuous coefficients and singular sources, SIAM J. Numer. Anal. 31, 1019-1044 (1994).

[19] Z. Li, A fast iterative algorithm for elliptic interface problems, SIAM J. Numer. Anal. 35, 230-254 (1998).

[20] Z. Li and K. Ito, The immersed interface method: Numerical solutions of PDEs involving interfaces and irregular domains, Frontiers in Applied Mathematics 33, SIAM (2006).

[21] Z. Li, T. Lin, Y. Lin and R. Rogers, An immersed finite element space and its approximation capability, Numer. Methods Partial Differential Equations 20, 338-367 (2004).

[22] Z. Li, T. Lin and X. Wu, New Cartesian grid methods for interface problems using the finite element formulation, Numer. Math. 96, 61-98 (2003).

[23] Z. Li, W. Wang, I. Chern and M. Lai, New formulations for interface problems in polar coordinates, SIAM J. Sci. Comput. 25, 224-245 (2003).

[24] T. Lin, Y. Lin and X. Zhang, Partially penalized immersed finite element methods for elliptic 
interface problems, SIAM J. Numer. Anal. 53, 1121-1144 (2015).

[25] T. Lin, Q. Yang and X. Zhang, Partially penalized immersed finite element methods for parabolic interface problems, Numer. Methods Partial Differential Equations 31, 1925-1947 (2015).

[26] T. Lin, Q. Yang and X. Zhang, A priori error estimates for some discontinuous Galerkin immersed finite element methods, J. Sci. Comput. 65, 875-894 (2015).

[27] X. Liu, R. Fedkiw and M. Kang, A boundary condition capturing method for Poisson's equation on irregular domains, J. Comput. Phys. 160, 151-178 (2000).

[28] X. Liu and T. Sideris, Convergence of the ghost fluid method for elliptic equations with interfaces, Math. Comput. 72, 1731-1746 (2003).

[29] R. Massjung, An unfitted discontinuous Galerkin method applied to elliptic interface problems, SIAM J. Numer. Anal. 50, 3134-3162 (2012).

[30] M. Oevermann and R. Klein, A Cartesian grid finite volume method for elliptic equations with variable coefficients and embedded interfaces, J. Comput. Phys. 219, 749-769 (2006).

[31] M. Oevermann, C. Scharfenberg and R. Klein, A sharp interface finite volume method for elliptic equations on Cartesian grids, J. Comput. Phys. 228, 5184-5206 (2009).

[32] C. Peskin, Numerical analysis of blood flow in the heart, J. Comput. Phys. 25, 220-252 (1977).

[33] R. Rangarajan and A. Lew, Parameterization of planar curves immersed in triangulations with application to finite elements, Int. J. Numer. Meth. Engng. 88, 556-585 (2011).

[34] Y. Shu, I. Chern and C. Chang, Accurate gradient approximation for complex interface problems in 3D by an improved coupling interface method, J. Comput. Phys. 275, 642-661 (2014).

[35] E. Wadbro, S. Zahedi, G. Kreiss and M. Berggren, A uniformly well-conditioned, unfitted Nitsche method for interface problems BIT Numer. Math. 53, 791-820 (2013).

[36] $\mathrm{H}$. Wu and Y. Xiao, An unfitted hp-interface penalty finite element method for elliptic interface problems, arXiv:1007.2893 (2010).

[37] W. Ying and J. Beale, A fast accurate boundary integral method for potentials on closely packed cells, Commun. Comput. Phys. 14, 1073-1093 (2012).

[38] W. Ying and C. Henriquez, A kernel-free boundary integral method for elliptic boundary value problems, J. Comput. Phys. 227, 1046-1074 (2007).

[39] W. Ying and W. Wang, A kernel-free boundary integral method for implicitly defined surfaces, J. Comput. Phys. 252, 606-624 (2013).

[40] S. Yu, Y. Zhou and G. Wei, Matched interface and boundary (MIB) method for elliptic problems with sharp-edged interfaces, J. Comput. Phys. 224, 729-756 (2007).

[41] Y. Zhou, S. Zhao, M. Feig and G. Wei, High order matched interface and boundary method for elliptic equations with discontinuous coefficients and singular sources, J. Comput. Phys. 213, 1-30 (2006). 